\title{
Open-loop Spectrum Analyzer Integrated Circuit for Nanoresonator Sensing
}

\author{
Hyunwoo Heo, ${ }^{1}$ Kwonsang Han, ${ }^{1}$ Hyungseup Kim, ${ }^{1}$ Jaesung Kim, ${ }^{1}$ Donggeun You, ${ }^{1}$ \\ Yongsu Kwon, ${ }^{1}$ Hyunjoong Lee, ${ }^{2}$ Yil suk Yang, ${ }^{2}$ and Hyoungho $\mathrm{Ko}^{1 *}$ \\ ${ }^{1}$ Department of Electronic Engineering, Chungnam National University, Daejeon 34134, Republic of Korea \\ ${ }^{2}$ Electronics and Telecommunications Research Institute (ETRI), Deajeon 34129, Republic of Korea
}

(Received April 16, 2019; accepted March 5, 2020)

Keywords: on-chip spectrum analyzer, nanoresonator, motional resistance, Butterworth-Van Dyke equivalent circuit, resistive sensitivity, lumped-parameter model

A nanoresonator can be applied as a biochemical sensor based on the change in the mass of the nanoresonator, and the mass change can be interpreted in the frequency domain. In this paper, we present an open-loop spectrum analyzer integrated circuit (IC) for a nanoresonator. The circuit determines the frequency characteristics of a nanoresonator, such as quality factor (Q-factor) and resonant frequency, and detects minute resistance changes of the nanoresonator that result in changes in Q-factor or resonant frequency. The proposed nanoresonator driver circuit is implemented using an open-loop system, and to characterize the open-loop frequency response of the nanoresonator, the IC includes a voltage-controlled oscillator, a transimpedance amplifier, and a 16-bit delta-sigma analog-to-digital converter. To compensate for the parasitic components that cause the distortions of the phase and magnitude response, a shunt-capacitance cancelling amplifier is used to cancel the effect of the shunt-parasitic capacitance of the nanoresonator. The simulated target nanoresonator is modeled using the Butterworth-Van Dyke equivalent circuit model with a resonant frequency of $10 \mathrm{MHz}$. The proposed nanoresonator driver circuit is fabricated using a standard $0.18 \mu \mathrm{m}$ complementary metal oxide semiconductor process with an active area of $2.346 \mathrm{~mm}^{2}$. The simulated resistive sensitivity of the IC is $5.1 \mathrm{mV} / \mathrm{k} \Omega$.

\section{Introduction}

As the nano/microelectromechanical system (NEMS/MEMS) sensor market continues to grow, a nanotechnology-based nanoresonator is expected to have various applications in the future, centering on the high-sensitivity sensor market. It can be applied as a biochemical sensor that is targeted for detecting protein, ${ }^{(1)}$ the density or viscosity of the surrounding liquid, or DNA. (2) The surrounding medium, DNA adsorption, and so forth cause the change in the damping parameter or mass in the equivalent mechanical lumped-parameter model. In the equivalent electrical Butterworth-Van Dyke model ${ }^{(3-6)}$ that consists of a motional branch 
(series resistance, capacitance, and inductance) and parallel static capacitance, the damper in the mechanical model acts as a dissipative element and is equivalent to the resistance, and the mass is an inertial element and equivalent to the inductance. The resonator driving circuit that reads out the motional resistance or inductance change can be applied to resonator-based biochemical sensors. In this paper, we focus on the motional resistance readout method for the detection of biochemical properties, such as the density and viscosity of fluid, ${ }^{(7-11)}$ or blood glucose.

The following are the two main systems for driving a resonator: open-loop and closed-loop systems. Several recent studies of a resonator driving integrated circuit (IC) using a closedloop system have been reported. ${ }^{(12-19)}$ In a closed-loop system using self-sustained oscillation and the resonant frequency tracking method, ${ }^{(12-14,16-18)}$ it is difficult to oscillate at an accurate resonant frequency. A resonator is a mechanical bandpass filter and if the resonator has a low Q-factor, the difference between the passband and the attenuated band is not obvious, so the frequency can be locked at an unwanted frequency. The parasitic capacitance of the resonator causes the distortions of the magnitude and phase response, and also makes the locking of the frequency at a resonant frequency difficult. Therefore, in order to develop a miniaturized highprecision nanoresonator through the NEMS process, the distortions of the phase and magnitude response caused by the effect of parasitic components ${ }^{(20,21)}$ should be adjusted.

In the closed-loop system, the quality factor (Q-factor) is indirectly measured by automatic gain control. On the other hand, in the open-loop system, the resonant frequency and Q-factor can be obtained from a full frequency response curve, but only in the case of a low Q-factor or multiple resonant frequencies. ${ }^{(5)}$ As with the closed-loop system, it is still important to cancel the parasitic capacitance in the open-loop system to alleviate the frequency response distortion and achieve a high Q-factor. ${ }^{(12)}$

Studies of a resonator driving IC for the determination of the resonator frequency response using the open-loop system have been rarely conducted. In this paper, an open-loop onchip spectrum analyzer IC for a nanoresonator is presented. The IC determines the resonant frequency and Q-factor from the full frequency response curve of the resonator. The shunt capacitance cancelling amplifier is used to cancel the effect of the parasitic shunt capacitance. As shown in Fig. 1, the proposed circuit is fully integrated into a single chip and implanted into the human body, which can be applied as a biochemical sensor for the continuous monitoring of blood glucose and blood pressure.

In Sect. 2, the equivalent mechanical/electrical resonator models of biochemical sensors are presented, and the proposed spectrum analyzer scheme for a nanoresonator and the operation principle are addressed. The simulation and experimental results are presented in Sect. 3. Finally, the conclusions are summarized in Sect. 4.

\section{Proposed Open-loop Spectrum Analyzer IC for Nanoresonator}

\subsection{Resonator model}

The equivalent mechanical and electrical resonator models are shown in Fig. $2 .^{(3)}$ In the mechanical model, protein or DNA adsorption results in the changes in mass and resonant 


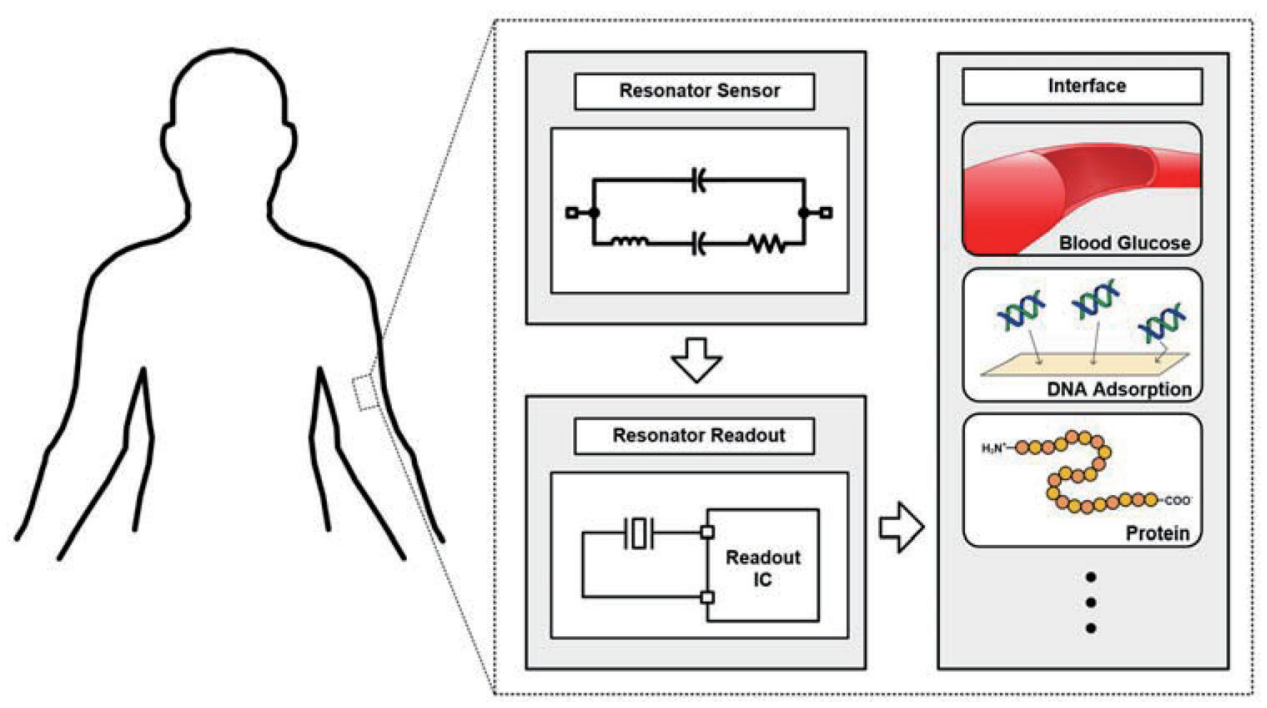

Fig. 1. (Color online) Fully implantable IC in human body.

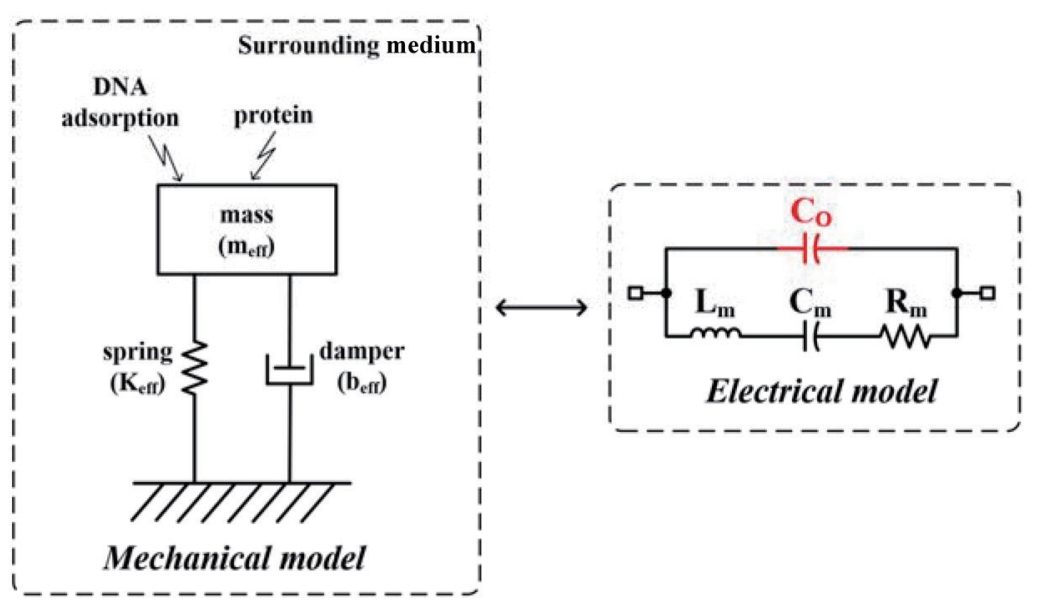

Fig. 2. (Color online) Equivalent mechanical and electrical resonator models.

frequency. Damping parameter changes with the state of the surrounding medium, which in turn changes the Q-factor. The damping parameter is equivalent to the motional resistance in the equivalent electrical model and the mass to the inductance so that they are equivalent to the changes in resistance and inductance in the electrical model of the resonator. The density and viscosity of the surrounding liquid or target materials can be detected by sensing the change in motional resistance or inductance.

In the equivalent electrical model, the motional series resonant frequency (MSRF) at which the effect of the parasitic capacitance $C_{O}$ is ignored is expressed as

$$
\omega_{r s}=\frac{1}{\sqrt{L_{m} C_{m}}}
$$


and the parallel resonant frequency that considers the parasitic capacitance $C_{O}$ is approximately expressed as

$$
\omega_{r p}=\sqrt{\frac{1}{L_{m} C_{m}}+\frac{1}{L_{m} C_{O}}-\frac{R_{m}{ }^{2}}{L_{m}{ }^{2}}} .
$$

In the case of $C_{O} \gg C_{m}$, Eq. (2) is approximated as ${ }^{(3)}$

$$
\omega_{r p}=\omega_{r s} \cdot \sqrt{1+\frac{C_{m}}{C_{O}}} .
$$

According to Eqs. (1)-(3), as the parasitic capacitance affects the frequency characteristics of the resonator, it is necessary to cancel the effect of the shunt parasitic capacitance to achieve the exact resonant frequency and high Q-factor.

\subsection{Proposed architecture of open-loop spectrum analyzer}

Figure 3 shows the detailed architecture of the open-loop spectrum analyzer for the nanoresonator. As shown in Fig. 3, the target nanoresonator can be modeled by the Butterworth-Van Dyke equivalent circuit model with a resistance of $39 \mathrm{k} \Omega$, an inductance of $30.9 \mathrm{mH}$, a capacitance of $8.4 \mathrm{fF}$, and a shunt parasitic capacitance of $4.15 \mathrm{pF}$ with an MSRF of $10 \mathrm{MHz}$.

According to Eqs. (1)-(3), the shunt parasitic capacitance must be cancelled using the parasitic capacitance canceller in the proposed scheme. This canceller consists of the unit gain inverting amplifier and programmable compensation capacitor. The unit gain inverting amplifier forms the antiphase clock from the voltage-controlled oscillator (VCO) output clock. The VCO output clock is incorporated into the shunt parasitic capacitance of the resonator, and

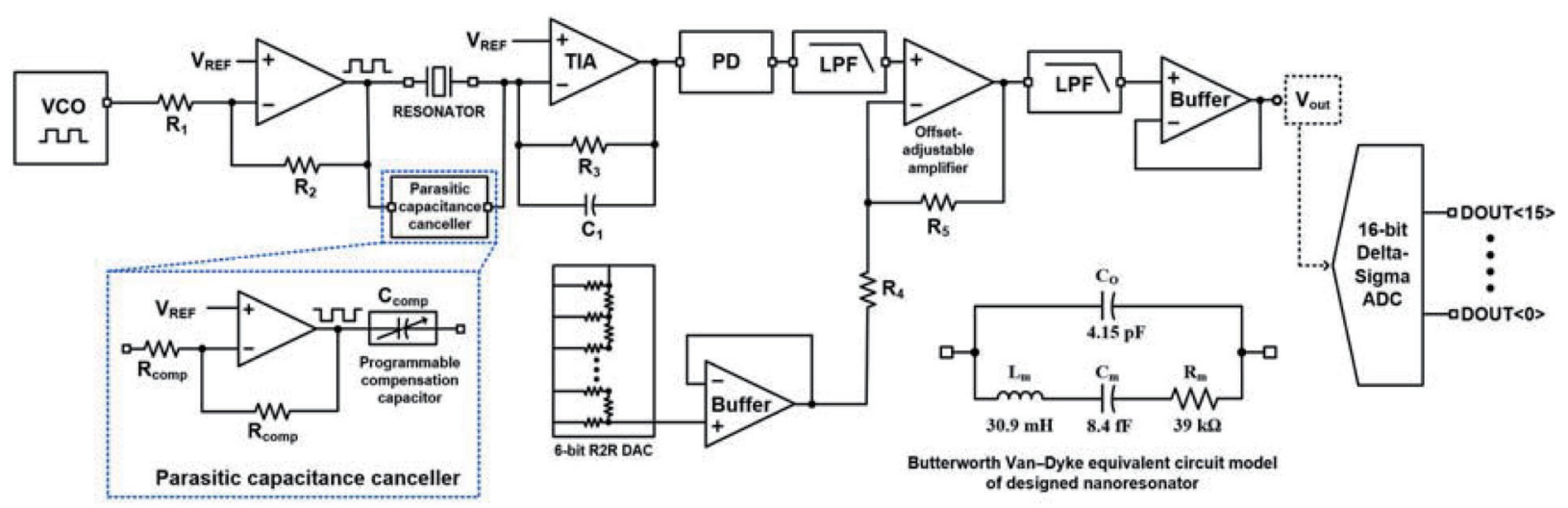

Fig. 3. (Color online) Proposed architecture of the open-loop spectrum analyzer. 
the antiphase clock from the VCO output is incorporated into the compensation capacitor. If the compensation capacitor is matched with the resonator with shunt parasitic capacitance, it acts on a minus-shunt parasitic capacitance and the parasitic capacitance can be canceled. The compensation capacitor is made programmable to compensate for the parasitic capacitance accurately because the shunt parasitic capacitance can be inaccurate.

The proposed spectrum analyzer IC includes the VCO and transimpedance amplifier (TIA) to characterize the open-loop frequency response of the nanoresonator. The VCO output frequency can be controlled from 3.7 to $22.3 \mathrm{MHz}$ so the frequency response curve of the resonator can be obtained by a frequency sweep using the VCO. The TIA output can be saturated by the amplification of the TIA and the amplitude of the VCO output clock is reduced using the under-unit gain inverting amplifier before driving the nanoresonator. The output current of the resonator driven by the VCO is incorporated into the TIA and converted into an amplified voltage. The output of the TIA is transformed to a DC peak voltage via the peak detector (PD), low-pass filter (LPF), and buffer.

At the resonant frequency, the series inductance $L_{m}$ and capacitance $C_{m}$ cancel each other out. Assume that the parasitic capacitance is canceled using the parasitic capacitance canceller, the gain from the input of the resonator to the output of the TIA at the resonant frequency can be expressed as

$$
A_{T I A}=-\frac{R_{3}}{R_{m}} \cdot \frac{1}{1+s C_{1} R_{3}}
$$

As shown in Eq. (4), the TIA output amplitude varies with the motional resistance of the resonator $\left(R_{m}\right)$, and the change in motional resistance can be detected from the peak voltage of the TIA output.

The difference in buffer output due to the resistance change can be unclear according to the PD output. The offset-adjustable amplifier makes the difference in output obvious according to the changes in resistance by adjusting the offset of the amplifier using an R2R digital-to-analog converter (DAC). The buffer output is converted into a digital code by the 16-bit delta-sigma analog-to-digital converter (ADC) and achieves a high resolution.

The schematic of the TIA is shown in Fig. 4. It has three amplification stages. For the implemented wide input range, $M_{1}$ and $M_{2}$ constitute the folded input stage and the cascode stage $M_{3}-M_{10}$ secondly amplifies the input signal. The output stage uses the Monticelli class AB consisting of $M_{11}-M_{16}$, achieving power efficiency. The Miller capacitor $C_{m}$ is used to compensate for the frequency response and ensure frequency stability. Figure 5 is the schematic of the PD. The same amplifier with the TIA is used for $A_{p}$. If the inverting input node $V_{i n}$ is higher than the non-inverting input node of the amplifier $A_{p}$, the output of $A_{p}$ is low. The transistor $M_{p}$ then turns on, $C_{p}$ is charged to the input voltage $V_{i n}$ and the output voltage $V_{\text {out }}$ follows $V_{\text {in }}$. If $V_{\text {in }}$ is lower than the non-inverting input node, $A_{p}$ outputs a high voltage and $M_{p}$ turns off, then $C_{p}$ is discharged through the resistor $R_{p}$. 


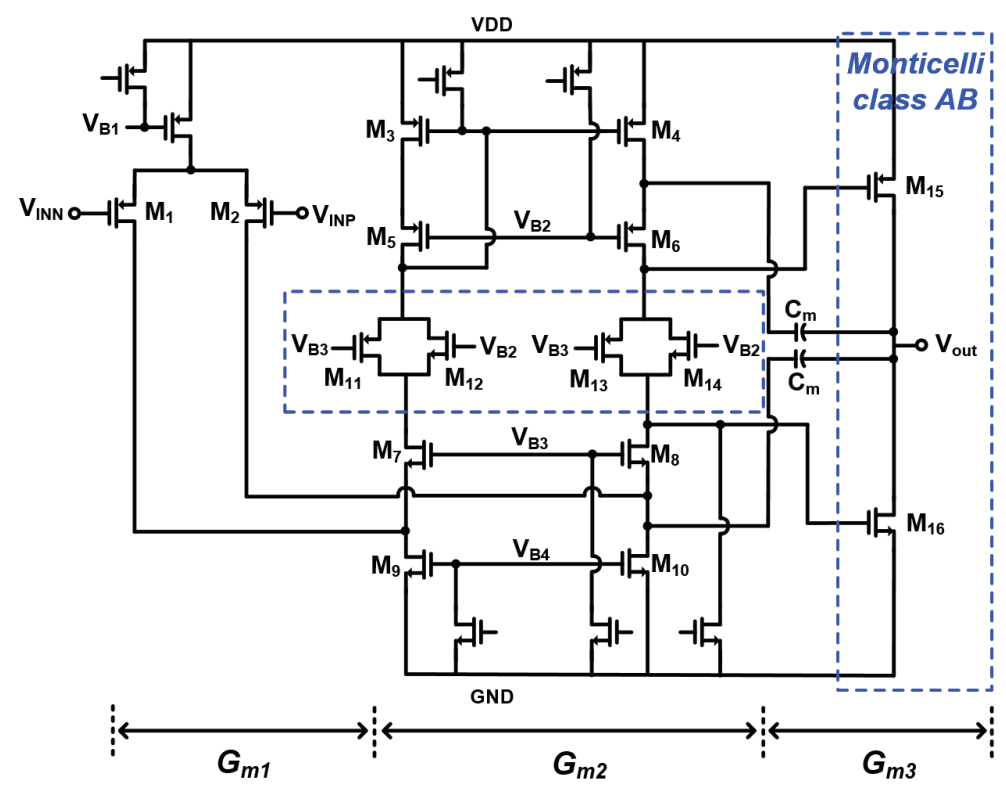

Fig. 4. (Color online) Schematic of TIA.

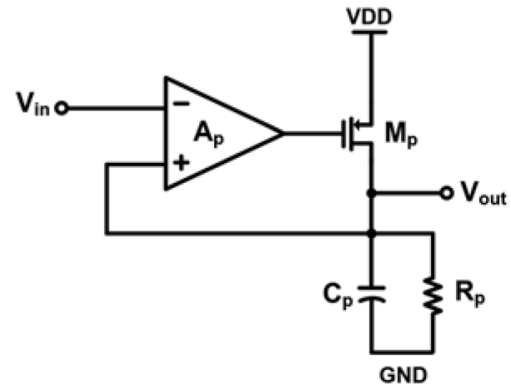

Fig. 5. Schematic of PD.

\section{Experimental Results}

\subsection{Simulation results}

The TIA input frequency is near the resonant frequency band, which is high in most cases, i.e., the bandwidth of the TIA should be sufficiently wide. Figure 6 shows the transfer function of the TIA. It has a DC gain of $80.5 \mathrm{~dB}$, and a unit-gain bandwidth of $84.95 \mathrm{MHz}$. Frequency stability is ensured with the phase margin of 77.78 degrees.

Figures 7(a) and 7(b) show the simulation results of motional resistance sensing. Figure 7(a) shows the AC simulation result obtained with the change in the $R_{m}$ of the nanoresonator model shown in Fig. 3. From the $R_{m}$ change of 37 to $41 \mathrm{k} \Omega$, the output voltage of the TIA changes by about $0.14 \mathrm{~dB}$ per $\mathrm{k} \Omega$. Under the same condition in the resistance change, the simulation result of the buffer output is shown in Fig. 7(b). The buffer output settles within $125 \mu$ s, and the output voltage change per $1 \mathrm{k} \Omega$ motional resistance change (resistive sensitivity) is about $5.1 \mathrm{mV} / \mathrm{k} \Omega$. 

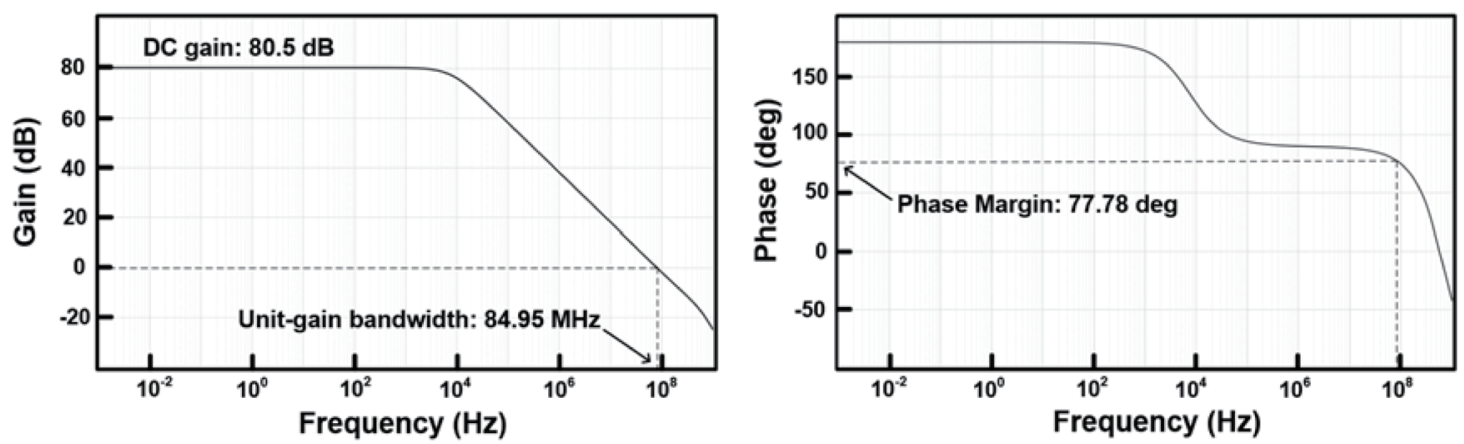

Fig. 6. Transfer function of TIA.

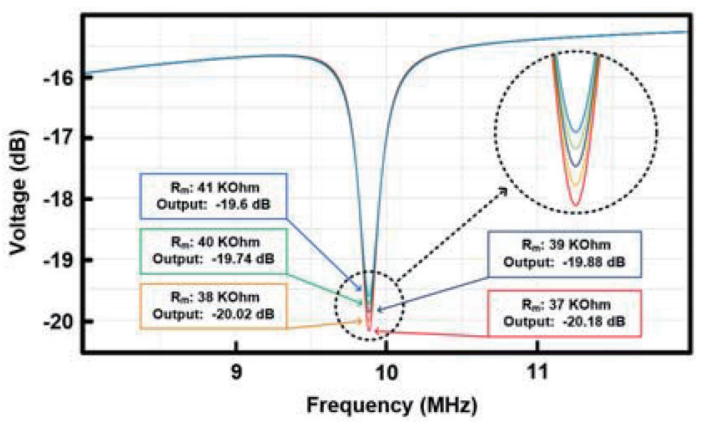

(a)

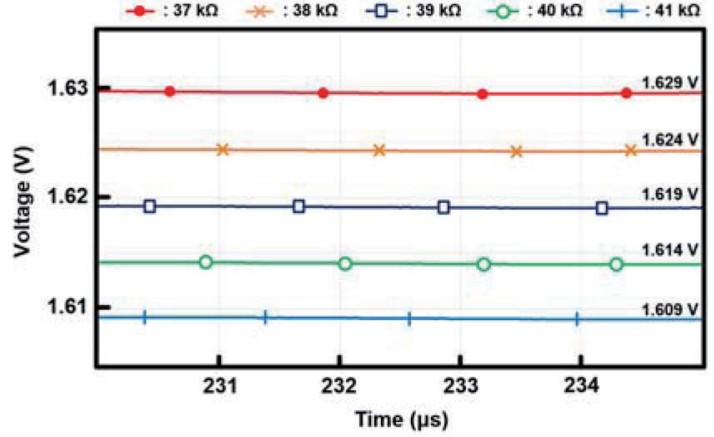

(b)

Fig. 7. (Color online) Simulation results under motional resistance $R_{m}$ changes. (a) Frequency response and (b) buffer output voltage.

\subsection{Measurement results}

Figure 8 is the die photograph of the proposed spectrum analyzer IC of the nanoresonator. The IC is designed using a complementary metal oxide semiconductor (CMOS) process with one $0.18 \mu \mathrm{m}$ polysilicon layer and six metal layers (1P6M) with an active area of $2.346 \mathrm{~mm}^{2}$.

Figures 9(a) and 9(b) show the results of the buffer output measurement using crystals in the frequency range of $\pm 2 \mathrm{kHz}$ at each resonant frequency. Commercial crystals with a shunt capacitance of about $7 \mathrm{pF}$ are used, and the programmable compensation capacitor array is adjusted to the shunt capacitance of the crystal. Figure 9(a) shows the measurement result of the frequency curve obtained using the crystal with a resonant frequency of $10 \mathrm{MHz}$. The measured resonant frequency is $9.9998 \mathrm{MHz}$, the cut-off frequencies are 9.99953 and 10.00028 MHz, and the Q-factor is 13421.48 . The result of the $6 \mathrm{MHz}$ crystal is shown in Fig. 9(b). The measured resonant frequency is $5.9998 \mathrm{MHz}$, the cut-off frequencies are 5.99961 and 6.0016 MHz, and the Q-factor is 10908.93 .

The measurement results of the PD output voltage noise and TIA input-referred current noise are shown in Figs. 10(a) and 10(b), respectively. As shown in Fig. 10(a), the output voltage noise 


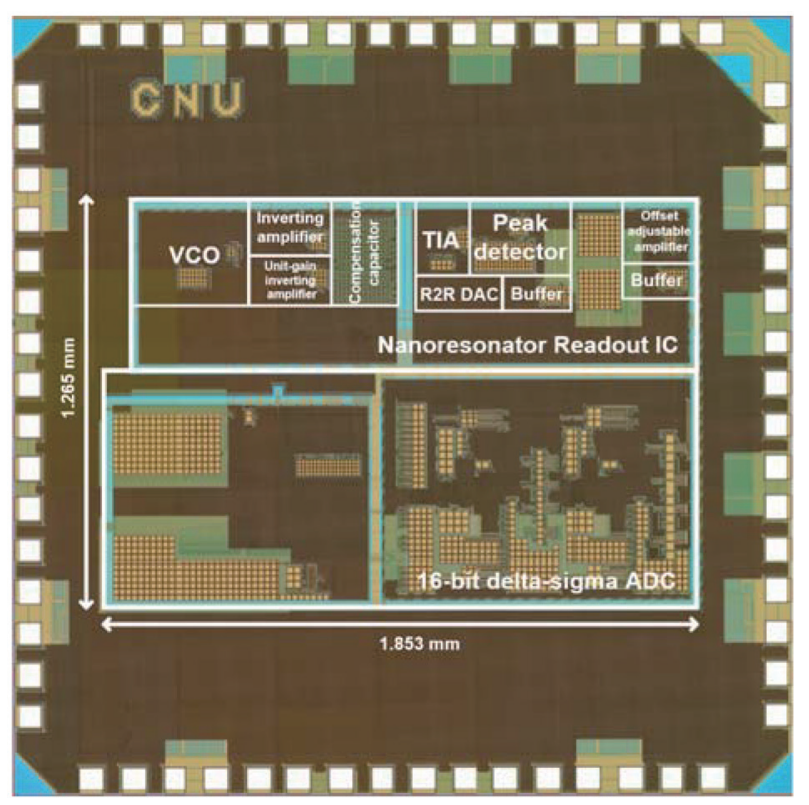

Fig. 8. (Color online) Die photograph of proposed open-loop spectrum analyzer IC.

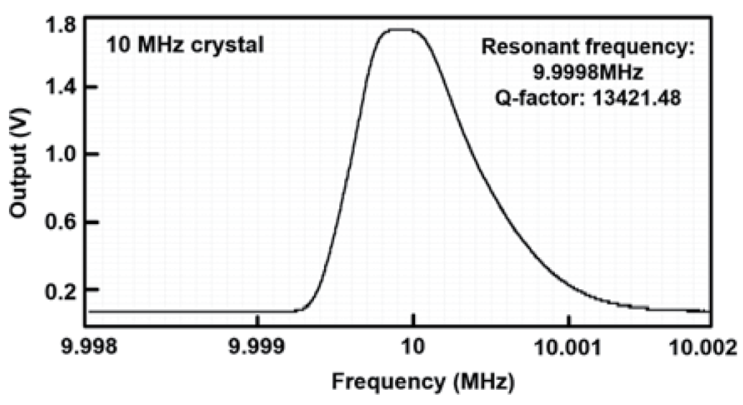

(a)

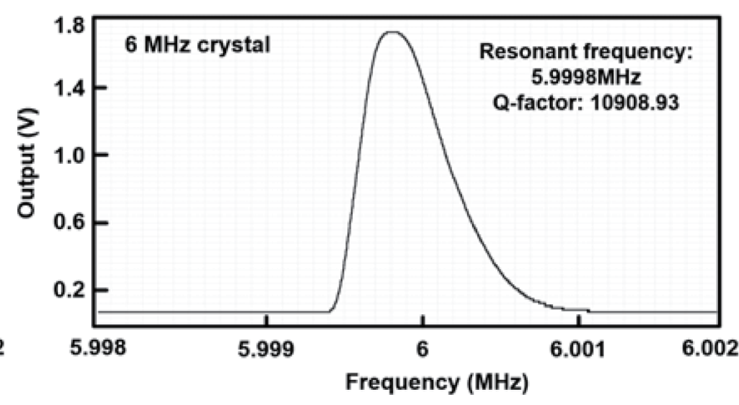

(b)

Fig. 9. Results of buffer output measurement using crystals with resonant frequencies of (a) 10 and (b) $6 \mathrm{MHz}$.

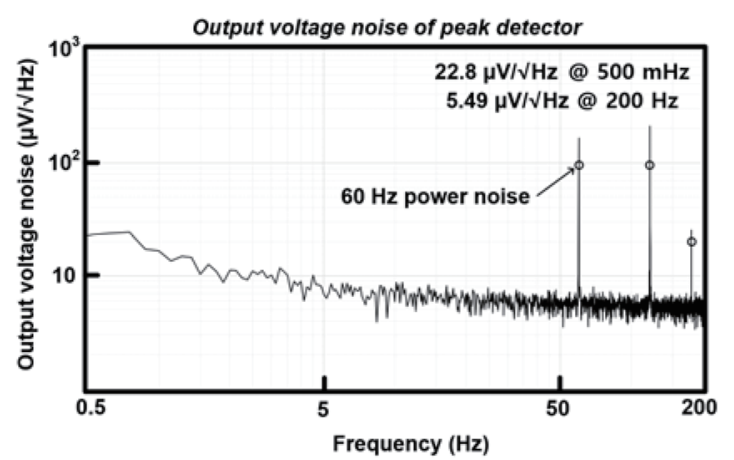

(a)

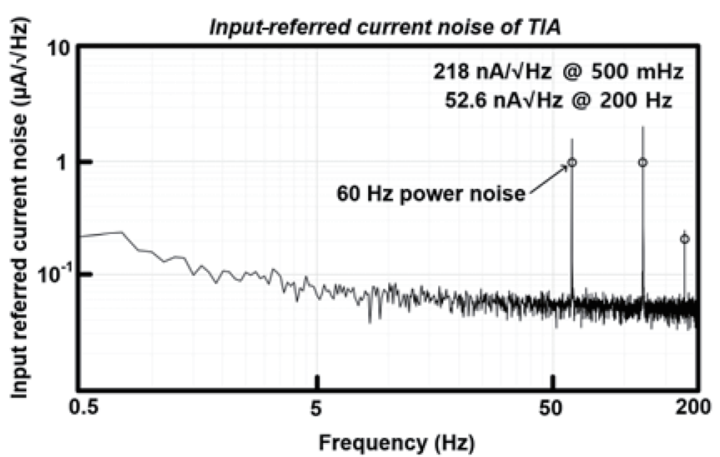

(b)

Fig. 10. Measurement results of (a) PD output voltage noise and (b) TIA input-referred current noise. 


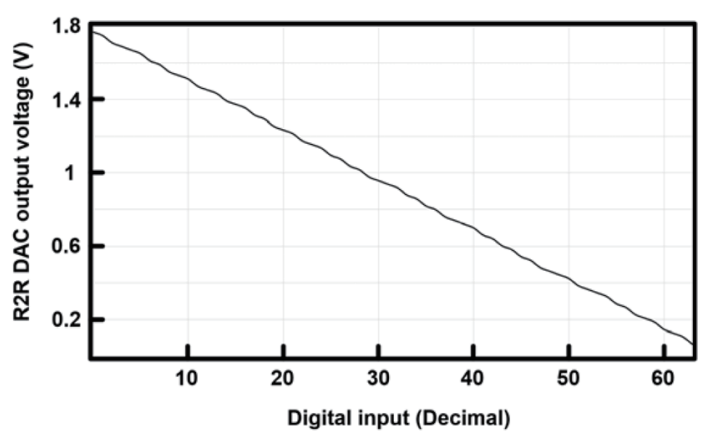

Fig. 11. Measurement results of 6-bit R2R DAC output according to digital input trim.

Table 1

Performance characteristics of proposed open-loop spectrum analyzer IC for nanoresonator.

\begin{tabular}{lcccc}
\hline Parameter & This work & Ref. 13 & Ref. 14 & Ref. 22 \\
\hline Technology $(\mu \mathrm{m})$ & 0.18 & 0.18 & 0.35 & 0.35 \\
Supply voltage $(\mathrm{V})$ & 1.8 & 2 & 2.5 & 7 \\
Power consumption $(\mathrm{mW})$ & 5.92 & 8.7 & 6.9 & 18 \\
Frequency $(\mathrm{MHz})$ & 10 & 8.29 & 20 & 25.6 \\
Q-factor & 13421 & 1040 & 160000 & 334 \\
Input referred current noise $(\mathrm{nA} / \sqrt{\mathrm{Hz}})$ & 52.6 & - & - & - \\
Resistive sensitivity $(\mathrm{mV} / \mathrm{k} \Omega)$ & 5.1 & - & - & - \\
Architecture & Open-loop & Closed-loop & Closed-loop & Closed-loop \\
Parasitic cancellation & Yes & No & No & No \\
Active area $\left(\mathrm{mm}^{2}\right)$ & 2.346 & 0.24 & 0.15 & 0.006 \\
\hline
\end{tabular}

is measured as $22.8 \mu \mathrm{V} / \sqrt{\mathrm{Hz}}$ at $500 \mathrm{mHz}$ and $5.49 \mu \mathrm{V} / \sqrt{\mathrm{Hz}}$ at $200 \mathrm{~Hz}$. Figure 10 (b) shows the TIA input-referred current noise. It is determined by dividing the feedback resistance of the TIA by the PD output voltage noise because the PD has unit gain. The TIA input-referred noise is measured as $218 \mathrm{nA} / \sqrt{\mathrm{Hz}}$ at $500 \mathrm{mHz}$ and $52.6 \mathrm{nA} / \sqrt{\mathrm{Hz}}$ at $200 \mathrm{~Hz}$.

The 6-bit R2R DAC output measurement results are shown in Fig. 11. As the digital input is trimmed from 0 to 63 in decimal, the R2R DAC output changes from 0.07 to $1.77 \mathrm{~V}$ in an almost linear manner. The output incorporated into the inverting input node of the offsetadjustable amplifier appropriately adjusts the offset. The performance characteristics of the proposed on-chip spectrum analyzer IC for the nanoresonator are shown in Table 1.

\section{Conclusions}

An open-loop on-chip spectrum analyzer IC for a nanoresonator is presented. The proposed IC is designed using a $0.18 \mu \mathrm{m} 1 \mathrm{P} 6 \mathrm{M}$ CMOS process with an active area of $2.346 \mathrm{~mm}^{2}$. The total power consumption of the IC is $5.92 \mathrm{~mW}$ with a $1.8 \mathrm{~V}$ supply. As shown in the measurement results obtained using crystals, the proposed IC operates as a spectrum analyzer of a nanoresonator that can obtain a full frequency curve and detect the frequency characteristics 
of the nanoresonator such as the resonant frequency and Q-factor. In the motional resistance change detection simulation, as shown in Figs. 7 and 8, the IC outputs different DC voltages with the change in motional resistance, and different frequency curves with different Q-factors or resonant frequencies can be obtained. The proposed IC can also detect the motional resistance change of the nanoresonator via these outputs and has a resistive sensitivity of about $5.1 \mathrm{mV} / \mathrm{k} \Omega$.

\section{Acknowledgments}

This work was supported by the Electronics and Telecommunications Research Institute (ETRI) Grant funded by the Korean government (20ZB1155, Development of Core Technologies for Implantable Active Devices). The EDA tool was supported by the IC Design Education Center (IDEC), Republic of Korea.

\section{References}

1 D. Li, S. Scarano, S. Lisi, P. Palladino, and M. Minunni: Sensors 18 (2018) 946. https://doi.org/10.3390/ s18040946

2 M. Pohanka: Materials 11 (2018) 448. https://doi.org/10.3390/ma11030448

3 Z. Yan and X. Zhou: Proc. 2010 IEEE Symposium on Piezoelectricity, Acoustic Waves and Device Applications (IEEE, 2010) 491. https://doi.org/10.1109/SPAWDA.2010.5744362

4 A. O. Niedermayer, T. Voglhuber-Brunnmaier, J. Sell, and B. Jakoby: Meas. Sci. Technol. 23 (2012) 085107. https://doi.org/10.1088/0957-0233/23/8/085107

5 H. Huang and D. Paramo: IEEE Trans. Ultrason. Ferroelectr. Freq. Control. 58 (2011) 2699. https://doi. org/10.1109/TUFFC.2011.2132

6 S. Giraud, S. Bila, M. Chatras, D. Cros, and M. Aubourg: Proc. 2009 IEEE Int. Frequency Control Symp. (IEEE, 2009) 890. https://doi.org/10.1109/TUFFC.2010.1378

7 T. Manzaneque, V. Ruiz-Diez, J. Hernando-Garcia, E. Wistrela, M. Kucera, U. Schmid, and J. L. SanchezRojas: Sens. Actuators, A 220 (2014) 305. https://doi.org/10.1016/j.sna.2014.10.002

8 E. Mehdizadeh, J. Gonzales, A. Rahafrooz, R. Abdolvand, and S. Pourkamali: In Tech. Dig. Hilton Head Workshop (2012) 359. http://citeseerx.ist.psu.edu/viewdoc/download?doi=10.1.1.725.2878\&rep=rep1\&type=pdf

9 J. Toledo, T. Manzaneque, V. Ruiz-Diez, M. Kucera, G. Pfusterschmied, E. Wistrela, U. Schmid, and J. L. Sanchez-Rojas: J. Micromech. Microeng. 26 (2014) 084012. https://doi.org/10.1088/0960-1317/26/8/084012

10 P. H. Ducrot, I. Dufour, L. Nicu, F. Mathieu, and C. Ayela: Proc. 2017 19th Int. Conf. Solid-State Sensors, Actuators and Microsystems (TRANSDUCERS) (IEEE, 2017) 1372. https://doi.org/10.1109/ TRANSDUCERS.2017.7994312

11 S. J. Martin, V. E. Granstaff, and G. C. Frye: Anal. Chem. 63 (1991) 2272. https://doi.org/10.1021/ac00020a015

12 H. Kim, B. Lee, Y. Ko, Y. Mun, Y. Kim, H. Lee, and H. Ko: Microsyst. Technol. 25 (2019) 2041. https://doi. org $/ 10.1007 / \mathrm{s} 0054$

13 F. Nabki and M. N. El-Gamal: Proc. 2008 IEEE 34th European Solid-State Circuits Conf. (IEEE, 2008$) 454$. https://doi.org/10.1109/ESSCIRC.2008.4681890

14 S. Seth, S. Wang, T. Kenny, and B. Murmann: Proc. 2012 IEEE European Solid-State Circuits Conf. (IEEE, 2012) 249. https://doi.org/10.1109/ESSCIRC.2012.6341332

15 J. Jahng, M. Lee, H. Noh, Y. Seo, and W. Jhe: Appl. Phys. Lett. 91 (2007) 023103. https://doi. org/10.1063/1.2753112

16 R. Lucklum and F. Eichelbaum: Piezoelectric Sensors (Springer, Heidelberg, 2006) p. 3. https://doi. org/10.1007/5346_023

17 H. C. Li, S. H. Tseng, P. C. Huang, and M. S. C. Lu: J. Micromech. Microeng. 22 (2012) 055024. https://doi. org/10.1088/0960-1317/22/5/055024

18 Y. W. Lin, S. Lee, S. S. Li, Y. Xie, Z. Ren, and C. C. Nguyen: IEEE J. Solid-State Circuits 39 (2004) 2477. https://doi.org/10.1109/JSSC.2004.837086

19 J. Tamayo, A. D. L. Humphris, A. M. Malloy, and M. J. Miles: Ultramicroscopy 86 (2001) 167. https://doi. org/10.1016/S0304-3991(00)00082-6 
20 A. Arnau, T. Sogorb, and Y. Jimenez: Rev. Sci. Instrum. 73 (2002) 2724. https://doi.org/10.1063/1.1484254

21 A. Arnau, T. Sogorb, and Y. Jimenez: IEEE Trans. Ultrason. Ferroelectr. Freq. 48 (2001) 617. https://doi. org $/ 10.1109 / 58.911746$

22 E. Marigo, J. Verd, J. L. Lopez, A. Uranga, and N. Barniol: J. Micromech. Microeng. 23 (2013) 115018. https:// doi.org/10.1088/0960-1317/23/11/115018

\section{About the Authors}

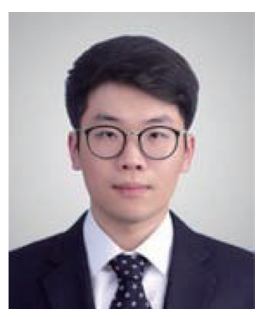

Hyunwoo Heo received his B.S. degree in electronics engineering from Chungnam National University, Daejeon, Republic of Korea, in 2019, where he is currently pursuing his M.S. degree. His current research interests are in the design of CMOS analog and mixed-mode integrated circuits.

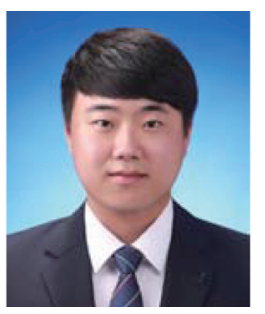

Kwonsang Han received his B.S. and M.S. degrees in electronics engineering from Chungnam National University, Daejeon, Republic of Korea, in 2018 and 2020, respectively. He is currently an engineer with Silicon Works Co., Ltd., Daejeon, Republic of Korea. His current research interests are in the design of CMOS analog and mixed-mode integrated circuits. His current research interests are in the design of CMOS analog and mixed-mode integrated circuits.

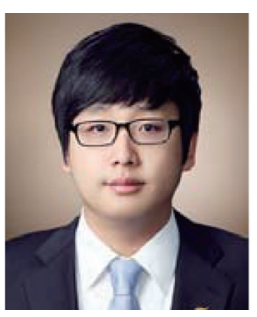

Hyungseup Kim received his B.S. degree in electronics engineering from Chungnam National University, Daejeon, Republic of Korea, in 2014, where he is currently pursuing his Ph.D. degree. His current research interests are in the design of sensor interface circuits, biosignal acquisition circuits, secure integrated circuits, data converters, and mixed-mode integrated circuits.

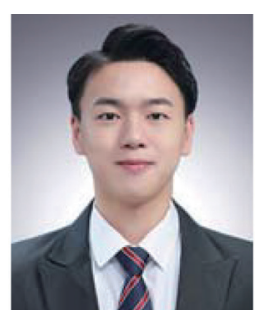

Jaesung Kim received his B.S. and M.S. degrees in electronics engineering from Chungnam National University, Daejeon, Republic of Korea, in 2018 and 2020, respectively. He is currently an engineer with Samsung Electronics Co., Ltd., Hwaseong, Republic of Korea. His current research interests are in the design of CMOS analog and mixed-mode integrated circuits.

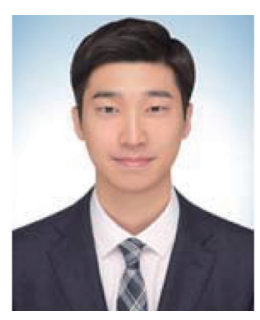

Donggeun You received his B.S. degree in electronics engineering from Chungnam National University, Daejeon, Republic of Korea, in 2019, where he is currently pursuing his M.S. degree. His current research interests are in the design of CMOS analog and mixed-mode integrated circuits. 


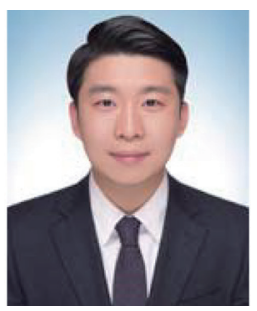

Yongsu Kwon received his B.S. degree in electronics engineering from Chungnam National University, Daejeon, Republic of Korea, in 2019, where he is currently pursuing his M.S. degree. His current research interests are in the design of CMOS analog and mixed-mode integrated circuits.

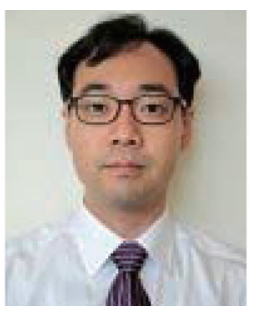

Hyunjoong Lee received his B.S., M.S., and Ph.D. degrees in electrical engineering and computer science from Seoul National University, Republic of Korea, in 2005, 2007, and 2012, respectively. He is currently a senior researcher with Electronics and Telecommunications Research Institute (ETRI), Republic of Korea. His current research interests include sensor interfaces for MEMS and bioapplications, and data converter and analog techniques in CMOS circuits.

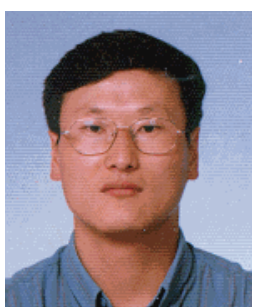

Yil suk Yang received his B.S., M.S. and Ph.D. degrees in school of electrical engineering and computer science from Kyungpook National University, Daegu, Korea, in 1989, 1994, and 2008, respectively. Before joining ETRI in 1999, he was with LG Semiconductor. Since 1999, he has worked at ETRI Basic Research Laboratory, where he has been engaged in the research of lowpower circuit design, high-energy-efficiency circuit design, and low-power microprocessor design.

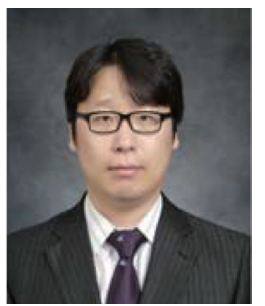

Hyoungho Ko received his B.S. and Ph.D. degrees in electrical engineering from Seoul National University, Republic of Korea, in 2003 and 2008, respectively. From 2008 to 2010, he worked with Samsung Electronics as a senior engineer. In 2010, he joined the Department of Electronics Engineering at Chungnam National University, Republic of Korea, where he is currently an associate professor. His current research interests are in the design of CMOS analog and mixed-mode integrated circuits. 